\title{
ESTRUCTURA MEDIÁTICA DE TIKTOK: ESTUDIO DE CASO DE LA RED SOCIAL DE LOS MÁS JÓVENES
}

\section{TIKTOK MEDIA STRUCTURE: CASE STUDY OF THE SOCIAL NETWORK OF THE YOUNGEST}

(D $\mathbb{R}^{\mathrm{a}}$ Manuel Antonio Conde del Rio. Universidad de Huelva. España.

\section{Cómo citar el artículo:}

Conde del Rio, M. A. (2021). Estructura mediática de tiktok: estudio de caso de la red social de los más jóvenes. Revista de Ciencias de la Comunicación e Información, 26, 59-77. http://doi.org/10.35742/rcci.2021.26.e126

\section{RESUMEN}

Los menores se han convertido, desde la aparición de TikTok en 2017, en creadores de contenido audiovisual de muy buena calidad, gracias a los smartphones y al software de edición y maquetación de vídeo de la propia plataforma. Aunque no solo es importante publicar buenos vídeos, pues los menores tienen que dominar otras competencias mediáticas para poder ofrecer un contenido de calidad y seguro. El objetivo de este trabajo ha sido actualizar los contenidos y conocer los cambios más destacados en la estructura mediática de TikTok y las competencias mediáticas de los menores en la misma. Se presenta una actualización de los contenidos y cambios más destacados en la estructura mediática de TikTok y las competencias mediáticas de los menores en la misma utilizando una metodología cualitativa con un estudio de caso de TikTok basado en los enfoques de análisis: comunicativo y competencial. A partir de este estudio de actualización se aportan nuevos datos sobre las acciones que se realizan en esta nueva red social sobre la alfabetización mediática y digital, continuando la apertura de este nuevo campo de investigación. Los resultados más destacados muestran la nueva estructura mediática de la red según sus posibilidades de interacción, la clasificación de acciones, de los vídeos y finalmente el análisis de la relación establecidas en la alfabetización mediática. Concluye afirmando los beneficios que suponen en el desarrollo competencial para los menores.

PALABRAS CLAVE: TikTok, Musical.ly, competencia mediática, red social, menores, smartphone

\section{ABSTRACT}

Youth people have become, since the appearance of TikTok in 2017, creators of very good quality audiovisual content, thanks to Smartphones and the platform's own video editing and layout software. Although it is not only important to publish good videos, since youth people have to master other media skills in order to offer quality and safe

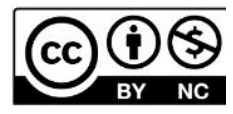

Recibido: 28/02/2021 - Aceptado: 09/06/2021 - Publicado: 28/06/2021 
content. The objective of this work has been to update the contents and learn about the most notable changes in the media structure of TikTok and the media competencies of youth people on it. An update of the contents and most notable changes in the media structure of TikTok and the media competencies of youth people in it is presented using a qualitative methodology with a TikTok case study based on the analytical approaches: communicative and competency. From this update study, new data is provided on the actions carried out in this new social network on media and digital literacy, continuing the opening of this new field of research. The most outstanding results show the new media structure of the network according to its possibilities of interaction, the classification of actions, of the videos, and finally the analysis of the relationship established in media literacy. It concludes by stating the benefits that they entail in the development of skills for minors.

KEYWORDS: TikTok, Musical.ly, media literacy, network, children, smartphone

\section{INTRODUCCIÓN}

Hoy en día, es una realidad que Internet y las Redes Sociales se han integrado de manera brillante en la vida cotidiana de la sociedad, siendo los jóvenes uno de los colectivos que más utilizan dichos instrumentos (Rostaminezhad et al., 2019), llegando a usarlos constantemente, destacando las Redes Sociales como una herramienta que les permite mantenerse en contacto con su. Grupo de iguales (Gutiérrez-Porlán et al., 2018).

Como consecuencia de esta realidad, ha podido demostrarse que el número de estudios alrededor de esta realidad se han visto incrementado año tras año (Fernández-de-la-Iglesia, 2020). Se aprecian, por una parte, un incremento en el número de investigaciones llevadas a cabo en España, las cuales pretenden identificar tanto la utilización formativa como social de las Redes Sociales (Gallardo et al., 2014; Gao \& Feng, 2016; García-Ruiz et al., 2016), así como el uso que se hace de los diferentes dispositivos (Gallardo et al., 2015), los modelos de utilización (Fernández, 2016), la dedicación que se realiza de las Redes Sociales (Gao \& Feng, 2016; Peña et al., 2018), las tácticas y dispositivos empleados por los jóvenes (Gutiérrez-Porlán et al., 2018) o la eficacia de las Redes Sociales en el ámbito académico (Rotaminezhad et al., 2018), entre otros. Por todo ello, este estudio de investigación pretende realizar un análisis de la Red Social TikTok, estableciendo, por un lado, las bases tanto comunicativas como competenciales relacionadas con esta plataforma, así como asentar los fundamentos necesarios para llevar a cabo futuras investigaciones relacionadas con esta Red Social tanto a nivel formativo como comunicativo.

\subsection{Las redes sociales y los menores}

Las Redes Sociales pueden parecer fenómenos recientes, pero ya desde la década de los 90 comenzaron a aparecer distintas plataformas sociales con el fin de crear perfiles personales y conectar estos con otros usuarios, los cuales pueden ser amigos y/o conocidos (Bargh \& McKenna, 2004; Boyd \&. Ellison, 2007; Kautz et al., 1997; Ros-Martín, 2009; Subrahmanyam et al., 2008). Desde el nacimiento de las primeras 
Redes Sociales estas han ido evolucionando y especializándose en distintos temas de interés para los usuarios, hasta llegar a la sectorización actual de estas, que han reconfigurado los mecanismos sociales de intercambio de la información (Domínguez, 2010; Gandsegui, 2011; Imaña, 2008; Orihuela-Colliva, 2008). Desde su aparición y durante la evolución de las mismas, los menores han sido protagonistas de ellas, con las ventajas y riesgos que esto conlleva para ellos. Este ha sido un tema muy recurrente en muchas investigaciones sobre los jóvenes y la incidencia de las Redes Sociales sobre ellos (Bernal-Bravo \& Anguloo, 2013; Bringué \& Sádaba-Chalezquer, 2011; Buckingham \&. Willet, 2013; Livingstone, 2002; Telefónica, 2009).

Siempre ha existido la presencia de menores en las Redes Sociales, pero la aparición de los Smartphones conectados a Internet como instrumentos de fácil acceso fue el momento de máxima presencia de estos en todas ellas, especialmente con el surgimiento de nuevas plataformas sociales enfocadas para este sector de la población (Corral, 2012; Hermosín-Mojeda \& Conde, 2015; Pedrero-Pérez et al., 2018; Tabernero et al., 2010).

YouTube, Instagram y Snapchat, desde su nacimiento, han acaparado a gran parte de los jóvenes que utilizan las Redes Sociales en todo el mundo. Estos espacios virtuales están perfectamente diseñados para atraer la atención de estos hacia ellos, espacios donde habitan y donde van adquiriendo competencias que los vuelven más exigentes con las mismas, tal y como se ha podido corroborar en muchas investigaciones (Castañeda-Quintero et al., 2011; Jiménez et al., 2016; Lavado, 2013; Marcelino-Mercedes, 2015). La creación de la plataforma de Musical.ly (2014) y su fusión posterior con TikTok (2017) es un ejemplo en los que los usuarios generan la necesidad de desarrollo de nuevas Redes Sociales que conecten con sus intereses.

\subsection{Investigaciones relacionadas con TikTok y Musical.ly}

En diversas búsquedas de investigaciones relacionadas con TikTok, antes Musical.ly, no aparecieron muchos resultados en las bases de datos, posiblemente por dos factores: la reciente creación de las mismas y que TikTok ha sido una Red Social de uso exclusivo para la población china hasta su fusión con Musica.ly. Los datos ofrecidos por las bases de bases de datos Web of Science (WOS), SCOPUS y Google Académico no fueron concluyentes, pero debemos entender que este nuevo fenómeno social posiblemente será objeto de estudio en los próximos años por lo que está suponiendo su irrupción en la población infantil y juvenil.

Antes de junio de 2020, en la base de datos de WOS, existían seis investigaciones indexadas donde Tiktok era parte del tema de estudio (Lim et al., 2019; Stokel-Walker, 2019), y de cuatro investigaciones de Musical.ly (Literat \&. Kligler-Vilenchik, 2019; Pérez-Escoda \& Contreras-Pulido, 2018, p.289). No es hasta 2017 cuando aparece la primera publicación en WOS (Rettberg, 2017, p.1). En Google Académico la búsqueda para TikTok antes de junio de 2020 fue de 46 publicaciones relacionadas con Tiktok y de 8 para Musical.ly, destacando entre ellas las de Davis, (2019) o Zhou (2019). La mayoría de las publicaciones existentes sobre TikTok eran de origen asiático. Tras la declaración de pandemia por COVID-19 en marzo de 2020 el uso de TikTok creció de forma exponencial, lo que llevó a muchas revistas a hacer especiales sobre el COVID19 que atrajo a muchos investigadores a publicar artículos relacionados con el 
fenómeno de la nueva red social durante este periodo (Becerra-Chauca \& TaypeRondan, 2020; Ballesteros-Herencia, 2020; Olivares-García \& Méndez-Majuelos, 2020).

\subsection{De Musical.ly a TikTok}

Musical.ly nació en 2014 y en poco tiempo el número de usuarios creció de forma muy importante. Alex Hofmann, presidente de operaciones Musical.Ly, llegó a afirmar que, si se comparaba el crecimiento de esta al de Facebook o Twitter, el de Musical.Ly fue mucho más rápido que el de ambos. Al igual que el número de usuarios, el volumen de negocios también creció rápidamente. Los datos del número de usuarios, el número de compañías y perspectiva de futuro, creación de sus propios influencers o muser gracias al programa de monetización y, finalmente, por el número creciente de descargas, posicionó a Musical.ly como la primera red de vídeos de sincronización de labios en Estados Unidos y en muchos países occidentales (Roobehmed, 2017). Como señalaron Pérez-Escoda \& Contreras-Pulido, Musica.ly despertaba "el interés científico y académico, en cuanto a su estructura y sus posibilidades en términos comunicativos y competenciales" (2018, p.215).

Asimismo, en China existía una aplicación similar llamada Douyin; sin embargo, fuera de este país, esta era denominada TikTok. Dicha Red Social era muy popular, cerca del 14\% de usuarios chinos la tenían en 2018 descargada en su Smartphone (Lim et al,m 2019; Xataka, 2018). En noviembre de 2017, TikTok compró Musical.ly por mil millones de dólares y se fusionaron ambas en una sola aplicación denominada TikTok. Con esto se situó como líder en solitario en este formato de Redes Sociales con más de 500 millones de usuarios en todo el mundo, convirtiéndose en una red que se podía visualizar en Occidente y China al mismo tiempo (Xataka, 2018, BBC Mundo, 2018; Chan, 2019; Wang, 2019) Kemp (2019) situó a TikTok como la cuarta aplicación (app) más descargada tras WhatsApp, Facebook Messenger e Instagram, superando a YouTube, Snapchat y Facebook. Esta Red Social se convirtió en un fenómeno viral en España y en todo el mundo, acumulando en 2019 más de 500 millones de usuarios o tiktokers, disponible en 150 mercados distintos y en 75 idiomas (Solís, 2019).

\subsection{El funcionamiento de TikTok y los tiktokers}

TikTok es una Red Social de vídeos cortos y transmisiones en directo con una duración máxima de 15 segundos (Yang et al., 2019, p.340). En ella se crean vídeos de formato corto tipo playback con una música de fondo donde los usuarios acostumbran a realizar bailes. La creación de vídeos cortos y sencillos fue una de las ideas que hicieron atractiva a la nueva plataforma de vídeos y fomentó de manera particular la creatividad de los usuarios (Sotelo, 2018).

La aplicación tiene bastantes funcionalidades para la creación de vídeos y con una usabilidad muy sencilla. Los contenidos pueden desarrollarse utilizando distintas formas de elaboración de vídeos como la cámara lenta, rápida, normal o emulando un timelapse. Se puede utilizar también la cámara frontal, como en Snapchat, o MSQRD para grabar vídeo en formato selfie (Santos, 2016), es decir, la aplicación ofrece muchas oportunidades de grabación de vídeos amateur a los usuarios, incluso con la posibilidad de grabar varios cortes para formar un solo vídeo, incluirles filtros $u$ otras 
posibilidades que hacen que aumente la dificultad de edición de vídeo, pero a su vez aumenta la calidad creativa de los mismos (Liqian, 2018).

El diseño de contenido de vídeo en las Redes Sociales es cada vez más sencillo y gracias a la popularidad de estos en TikTok, el resto de Redes han implementado las mismas funciones; así lo afirma Wang al decir que "los vídeos móviles de formato corto en aplicaciones de redes sociales son cada vez más populares" (2020, p.106373). La grabación de vídeos con el Smartphone en primera persona y primer plano son muy persuasivos para el resto de usuarios, y esta es una de las peculiaridades más destacada de esta Red Social (Herrman, 2019; Wang, 2020).

Los desafíos o retos son unas de las características más destacadas de TikTok, así como que los usuarios se autodenominan tiktokers. Estas dos características juntas ayudan a que la interacción entre los usuarios sea muy efectiva y grande, lo cual favorece la difusión de los vídeos de forma muy viral, llegando a crear en muy poco tiempo muchos influencers en la Red que, a su vez, generan aún más contenido copiando el del resto de usuarios (Zhou, 2019; Ahlse et al., 2020). Es importante mencionar cómo los que más repercusión e influencia tienen entre sus seguidores llegan a encontrar una verdadera fuente de ingresos con los vídeos que editan y suben a sus cuentas, teniendo, del mismo modo, el respaldo de empresas que quieren publicitarse a través de estas cuentas, ya sea de manera directa o indirecta (Conde et al., 2020).

\subsection{Dimensiones de la competencia mediática}

Según Ferrés \& Piscitelli, "la competencia mediática comporta el dominio de conocimientos, destrezas y actitudes" (2012, p.79). Para analizar la competencia mediática existen seis dimensiones que conforman la misma: lenguajes, tecnología, procesos de interacción, procesos de producción y difusión, ideología y valores, y estética. Estas dimensiones tienen que ver en como las personas reciben los mensajes e interaccionan con ellos, así como en las personas producen mensajes (Ferrés \& Piscitelli, 2012).

Las dimensiones sobre la competencia mediática han sido utilizadas por muchos autores como base para sus estudios de investigación (Muñoz-Saldaña et al., 2011; Pérez-Rodríguez \& Delgado-Ponce, 2012; Aguaded-Gómez, 2012; Ferrés, 2013; Marín-Gutiérrez et al., 2013; Contreras-Pulido et al., 2014), el desarrollo de las mismas ayuda mucho a los investigadores para realizar los análisis necesarios sobre estas. Según Ferrés \& Piscitelli (2012), las personas tenían que tener una serie de capacidades o conocimientos, los cuales estaban desarrollados de forma detallada en las seis dimensiones en los dos ámbitos: análisis y expresión.

Rettberg (2017, p.1) indicaba que los gestos eran elementos importantes en la comunicación no verbal y estos cobraban gran importancia en la competencia de los menores al utilizarlos como forma de comunicación codificada en los vídeos que suben a la plataforma musical. Los menores subían vídeos de 15 segundos donde no solo seleccionaban la música y la sincronización de los labios, pues los mismos, con mucha frecuencia, incluían una sucesión de signos con las manos que mejoraban el 
mensaje de sincronización de los labios y que este evolucionaba para crear un nuevo sistema de códigos conocidos por los usuarios de la plataforma.

Pérez-Escoda \& Contreras-Pulido (2018), tras analizar Musical.ly, Ilegaron a la conclusión de que el uso de esta propiciaba competencias mediáticas y diseñaron la estructura mediática de la misma.

\section{OBJETIVOS}

Para llevar a cabo este estudio, se ha pretendido conocer los cambios más destacados de la estructura mediática de TikTok, estableciendo una relación con las competencias mediáticas que se desarrollan entre los menores. Se establecieron, del mismo, una serie de objetivos específicos para abordar con mayor profundidad este estudio, siendo estos los siguientes:

- Conocer la estructura mediática de la Red Social, así como su grado de interacción.

- Establecer una clasificación de las acciones.

- Identificar las áreas competenciales y sub competenciales de la alfabetización mediática, estableciendo una serie de relaciones entre ellas y las acciones llevadas a cabo.

\section{DISEÑO Y METODOLOGÍA DE LA INVESTIGACIÓN}

Dentro del ecosistema audiovisual actual en el que la sociedad se encuentra inmersa, donde la utilización de dispositivos móviles y de las Redes Sociales es elevado, se ha contemplado la realización de un estudio de caso de la Red Social TikTok, siendo los niños y adolescentes el mayor colectivo con cuentas en ella a nivel mundial.

En relación a la metodología empleada en la presente investigación es de carácter cualitativo, asentando el estudio sobre dos enfoques, uno de carácter comunicativo, donde se lleva a cabo un estudio sobre la comunicación en TikTok, según la teoría de los dos escalones de la comunicación de Katz \& Lazarsfeld (Rodrigo-Alsina, 2001), el cual se fundamenta sobre el análisis de la comunicación interpersonal de la comunicación mediada, así como de la repercusión a nivel personal del individuo que expresa su punto de vista a sus seguidores, para lo cual se realiza un análisis de aquellos perfiles más populares atendiendo a una serie de variables de estudio, tales como cantidad de fans, me gustas y comentarios en los últimos vídeos, etiquetas del perfil y, por último, perfil sociodemográfico relativos a la edad y al género. El segundo nivel de análisis hace referencia al enfoque competencial, el cual se asienta sobre la teoría de los componentes de la comunicación mediada de Katz, Rice \& Acord (2004) así como de sus variables asociadas, mediante el cual se va a favorecer que se asienten las bases comparativas que correlacionen las actividades que ofrece TikTok con las destrezas mediáticas y digitales asentadas con anterioridad, con el fin de revelar como se promociona a través de esta red estas competencias de una manera informal. A través de los datos que se han conseguido al realizar estos dos análisis, se aporta información importante en lo que a la comunicación y a las competencias se refiere, lo cual va a favorecer la mejor comprensión de las oportunidades que las Redes Sociales brindan a los más jóvenes. 
El análisis previo de Pérez-Escoda \& Contreras-Pulido (2018, p.289) permite, asimismo, actualizar los contenidos, conocer los cambios más destacados sobre TikTok, y saber cómo evolucionan los datos más relevantes en clave comunicativa y competencial, que ayudan a comprender las posibilidades de desarrollo de las competencias mediáticas en los menores en relación con las dimensiones de la competencia mediática (Ferrés \& Piscitelli, 2012).

\section{RESULTADOS}

Para comenzar con el análisis de resultados, se quiere conocer la Red Social TikTok a nivel comunicativo, para lo cual se toma en consideración la teoría de los dos escalones de la comunicación de Katz \& Lazarsfel (Rodrigo-Alsina, 2001), donde se llevará cabo un estudio sobre la estructura mediática de dicha red, dando a conocer sus oportunidades de relación con los usuarios.

\subsection{Estructura mediática de TikTok}

TikTok, en 2019, se convirtió en una plataforma utilizada de forma más habitual por los menores entre los 11 y los 16 años, que conforma el mayor grueso de usuarios y que tenía alrededor de 500 millones de usuarios, de los cuales un $70 \%$ eran de género femenino. De estas cifras, concretamente las que aluden a las niñas que utilizan TikTok, hacen que sea necesario realizar estudios y análisis sobre el uso de las mismas y como este entorno está influenciando al desarrollo de su competencia mediática (Smith, 2019).

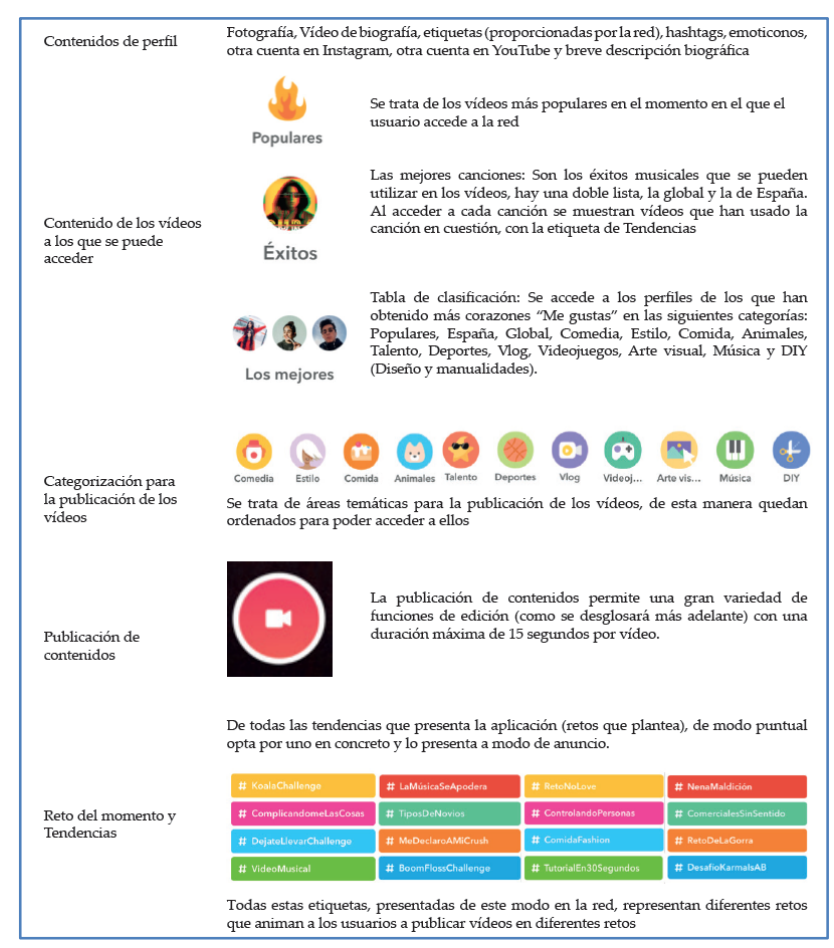

Figura 1: Estructura mediática de la red Musical.ly según las posibilidades de interacción respecto a las variables estudiadas

Fuente: (Pérez-Escoda \& Contreras-Pulido, 2018, p. 286) 
Se observan las posibilidades que los usuarios tenían para interactuar en Musical.ly (Figura 1), es decir, contenido de perfil, contenido de los vídeos a los que puede acceder, categorización para la publicación de los vídeos, publicación de contenidos, así como el reto del momento y tendencias. La estructura mediática desarrollada inicialmente (Figura 1), y tras una profunda actualización, presentó diversas modificaciones y pudo servir para crear una nueva relación de la estructura mediática de TikTok (Tabla 1) y poder, así, comprender mejor las posibilidades de interacción de la misma.

Tabla 1: Evolución en la estructura mediática de TikTok según las posibilidades de interacción respecto a las variables estudiadas

\begin{tabular}{|c|c|c|}
\hline \multicolumn{2}{|c|}{ Variables } & $\begin{array}{c}\text { Descripción } \\
\text { El perfil contiene la fotografía y el vídeo del } \\
\text { mismo, una descripción corta del muser y } \\
\text { enlaces a Instagram y/o YouTube. }\end{array}$ \\
\hline $\begin{array}{c}\text { Contenidos del perfil } \\
\text { Contenido de los vídeos } \\
\text { a los que se puede } \\
\text { acceder }\end{array}$ & $\begin{array}{c}\text { Dos apartados: } \\
\text { "Siguiendo", muestra vídeos de muser a los que seguimos. } \\
\text { "Para ti", muestra vídeos de muser que nos pueden interesar } \\
\text { en función a los vídeos que hemos estado visualizando. }\end{array}$ \\
\hline $\begin{array}{c}\text { Categorización para la } \\
\text { publicación de los vídeos }\end{array}$ & $\begin{array}{c}\text { No existe categorización de áreas temáticas para la publicación de } \\
\text { vídeos y fue eliminado por TikTok. }\end{array}$ \\
\hline $\begin{array}{c}\text { Publicación de } \\
\text { contenidos }\end{array}$ & $\begin{array}{c}\text { La publicación de contenidos tiene una gran variedad } \\
\text { de funciones. }\end{array}$ \\
\hline $\begin{array}{c}\text { Reto del momento y } \\
\text { tendencias }\end{array}$ & $\begin{array}{c}\text { TikTok cambión esta funcionalidad, y ahora, en el botón } \\
\text { "descubrir", aparecen los hashtags más populares que } \\
\text { agrupan a distintos vídeos. }\end{array}$ \\
\hline
\end{tabular}

Fuente: Elaboración propia

\subsection{Grado de interacción de los tiktokers}

A partir del análisis de la estructura mediática de TikTok, se realiza un análisis de las cuentas de los tiktokers con más éxito en la actualidad, con el objeto de observar en el contexto el grado de interacción a partir del número de fans y el número de corazones e interacciones en sus cuentas personales. 


\section{ESTRUCTURA MEDIÁTICA DE TIKTOK: ESTUDIO DE CASO DE LA RED SOCIAL DE LOS MÁS JÓVENES}

Tabla 2: Las cuentas de TikTok con más seguidores del mundo

\begin{tabular}{|c|c|c|c|c|c|}
\hline & Muser & Edad & Sexo & Millones Fans & № Corazones \\
\hline @charlidamelio & Charli D'Amelio & 16 & $\mathrm{M}$ & 85.4 & $6.4 \mathrm{~B}$ \\
\hline @addisonre & Addison Rae & 19 & $\mathrm{M}$ & $59.6 \mathrm{M}$ & $3.6 \mathrm{~B}$ \\
\hline @zachking & Zachary Michael King & 30 & $\mathrm{H}$ & $49.3 \mathrm{M}$ & $567.7 \mathrm{M}$ \\
\hline @lorengray & Loren Gray & 18 & $\mathrm{M}$ & $47.2 \mathrm{M}$ & $2.6 \mathrm{~B}$ \\
\hline @spencerx & Spencer X & 28 & $\mathrm{H}$ & $43.7 \mathrm{M}$ & $1.1 \mathrm{~B}$ \\
\hline @riyaz.14 & Riyaz Aly & 16 & $\mathrm{H}$ & $43.3 \mathrm{M}$ & $2 \mathrm{~B}$ \\
\hline @justmaiko & Michael Le & 20 & $\mathrm{H}$ & $39.0 \mathrm{M}$ & 1B \\
\hline @dixiedamelio & Dixie D’Amelio & 19 & $\mathrm{M}$ & $37.5 \mathrm{M}$ & $1.1 \mathrm{~B}$ \\
\hline @willsmith & Will Smith & 51 & $\bar{H}$ & $36.3 \mathrm{M}$ & $179.2 \mathrm{M}$ \\
\hline @jasonderulo & Jason Derulo & 30 & $\mathrm{H}$ & $34.8 \mathrm{M}$ & $735.8 \mathrm{M}$ \\
\hline @babyariel & Ariel Rebecca Martin & 19 & M & $34.7 \mathrm{M}$ & $1.9 \mathrm{~B}$ \\
\hline @brentrivera & Brent Rivera & 22 & $\mathrm{H}$ & $33.1 \mathrm{M}$ & 1B \\
\hline @mr_faisu_07 & Faisal Shaikh & 25 & $\mathrm{H}$ & $32.0 \mathrm{M}$ & $2.0 \mathrm{~B}$ \\
\hline @gilmehercroes & Gilmher Choes & 27 & $\mathrm{H}$ & $29.5 \mathrm{M}$ & $738.2 \mathrm{M}$ \\
\hline @_anshfakhan & Arishfa Khan & 17 & M & $28.5 \mathrm{M}$ & $1.1 \mathrm{~B}$ \\
\hline @nishaguragain & Nisha Gunagain & 22 & $\mathrm{M}$ & $28.0 \mathrm{M}$ & $755.8 \mathrm{M}$ \\
\hline @kristenhancher & Kristen Hancher & 21 & $\bar{M}$ & $24.6 \mathrm{M}$ & $1.7 \mathrm{~B}$ \\
\hline @jacobsartorius & Jacob Sartorius & 17 & $\mathrm{H}$ & $23.7 \mathrm{M}$ & $1.6 \mathrm{~B}$ \\
\hline @jiffpom & Jiffpom & 10 & PERRO & $21.2 \mathrm{M}$ & $516,2 \mathrm{M}$ \\
\hline
\end{tabular}

Fuente: Elaboración propia basada en fuentes (Influencer Marketinghub, 2020; Libretilla, 2020).

Entre los tiktokers con más seguidores del mundo existen un grado de interacción muy elevado, según los datos que se pueden observar sobre el número de seguidores, todos millonarios, y el número de corazones o me gustas que les dan a sus vídeos, presentando unas cifras billonarias (Tabla 2). Estas cifras, probablemente, arroja un corte de imagen de la interacción tan elevada que existe entre los usuarios de TikTok, pues si se hubieran escogido un corte de imagen de otra franja en la tabla, de igual forma, en función al número de seguidores, la interacción sigue los mismos patrones de comportamiento que en esta que hemos analizado.

\subsection{Clasificación de las acciones en TikTok}

Para poder correlacionar las acciones con la competencia mediática, se realiza un estudio comparativo entre Musical.ly y TikTok, el cual ha permitido establecer una nueva clasificación de acciones:

\section{A.- Relacionadas con el contenido del perfil:}

- Editar contenidos personales: nombre, vídeo y descripción corta.

- Añadir enlaces a Instagram y YouTube.

- Encontrar amigos buscando en contactos del Smartphone o Facebook.

- Invitar a amigos mediante mensaje de textos o correo electrónico.

- Cambiar la contraseña y región.

- Seguir a usuarios de TikTok en Instagram o Facebook. 
- Hacer vídeos de 15 segundos con música o de 1 minuto si es con la propia voz del muser. Diferenciar entre vídeo privado y público, hacer un vídeo privado, borrar y guardar vídeos en la galería.

- Cambiar la configuración de la privacidad:

- Configurar como cuenta privada.

- Permitir que otros puedan encontrar mi cuenta y esta sea sugerida.

- Personalizar los anuncios.

- Decidir quién puede publicar comentarios, reaccionar, hacer dúo o recibir mensajes.

- Permitir descarga.

- Decidir quién puede ver los vídeos a los que se le han dado corazón.

- Filtrar comentarios por palabras clave.

- Ver la lista de bloqueados.

- Cambiar la configuración general de la cuenta.

- Cambiar las push (nuevos seguidores, me gusta y comentarios)

- Elegir idioma, que permitirá personalizar la experiencia visual.

- Cambiar la desintoxicación digital:

- Gestionar el tiempo de pantalla.

- Modo restringido para filtrar el contenido inapropiado.

\section{Opciones eliminadas de Musical.Ly}

- No se exportó comprar camisetas de Musical.ly.

- Obtener ayuda para hacer duetos.

- Hacer copias de seguridad de los vídeos privados.

- Poder visitar los vídeos más populares.

\section{B.- Relacionadas con el contenido de vídeos a los que se puede acceder:}

- Ver los vídeos de los usuarios a los que sigues o los indicados para ti.

- Interactuar y comunicar directamente a través de corazones o con los usuarios a través de comentarios en los vídeos.

- Compartir el vídeo en diversas Redes Sociales: WhatsApp, Messenger, Instagram, Stories de Instagram, Facebook, Facebook Life, SMS, Twitter, Email, otro.

- Copiar enlace del vídeo.

- Acceder a la música que utiliza el vídeo para ver más vídeos que la han utilizado o grabar con la misma música.

\section{C.- Relacionadas con la publicación de contenidos:}

No es posible publicar vídeos relacionados con la categorización de TikTok, pero si de un tema accediendo desde "descubrir" al hashtag donde encontraremos relacionados con esa etiqueta.

\section{D.- Relacionadas con el reto del momento y tendencias:}

- No existe la opción de hacer los retos del momento.

- Las tendencias han cambiado su forma de visualización, ahora es "descubrir" hashtag 


\subsection{Competencias y sub competencias y las acciones en TikTok}

Se establecieron ocho áreas competenciales, las cuales presentaban otras áreas sub competenciales, que posibilitaban conformar la relación entre las mismas de la alfabetización mediática y TikTok (Tabla 3).

Tabla 3: Relación establecida entre áreas competenciales de la alfabetización mediática y acciones en TikTok

\begin{tabular}{|c|c|}
\hline Áreas & es que se engloban en \\
\hline $\begin{array}{l}\text { ACCESO: Información y } \\
\text { recepción }\end{array}$ & $\begin{array}{l}\text { - Añadir enlaces a cuentas de Instagram y YouTube } \\
\text { - Buscar amigos de los contactos del Smartphone o de Facebook } \\
\text { mediante mensaje de texto o email } \\
\text { - Comprar monedas para comprar regalos } \\
\text { - Hacer vídeos de } 15 \text { segundos con música o vídeos de un minuto } \\
\text { si es con la propia voz del muser, hacer un vídeo privado, etc. }\end{array}$ \\
\hline $\begin{array}{l}\text { MANEJO: Conocimiento } \\
\text { y uso del medio }\end{array}$ & $\begin{array}{l}\text { plicaciones } \\
\text { ña } \\
\text { dario para recuperar cuenta }\end{array}$ \\
\hline $\begin{array}{r}\text { CREACIÓN: } \\
\text { y este }\end{array}$ & $\begin{array}{l}\text { ón de la privacidad de la cuenta } \\
\text { general de la cuenta }\end{array}$ \\
\hline $\begin{array}{l}\text { COMUNICACl } \\
\text { Interacción y us } \\
\text { lenguaje }\end{array}$ & $\begin{array}{l}\text { - Editar contenidos, nombre, vídeo y descripción corta } \\
\text { - Invitar a amigos de los contactos del Smartphone o de Facebook } \\
\text { mediante mensaje de texto o email } \\
\text { - Comprar monedas para comprar regalos } \\
\text { - Cargar un vídeo de más de } 15 \text { segundos, grabar un vídeo sin } \\
\text { mantener presionado el botón de grabación, diferenciar entre } \\
\text { vídeo privado y público, hacer un vídeo privado, copia de } \\
\text { seguridad de vídeos, borrar vídeos, guardar en la galería. } \\
\text { - Interactuar con vídeos mediante corazones y comunicar con } \\
\text { muser mediante comentarios en los vídeos. }\end{array}$ \\
\hline $\begin{array}{r}\text { CREACIÓN: } \\
\text { y est }\end{array}$ & $\begin{array}{l}\text { - Compartir vídeos en diversas Redes Sociales: WhatsApp, } \\
\text { Messenger, Instagram, Stories de Instagram, Facebook, } \\
\text { Facebook Life, SMS, Twitter, Email, otro } \\
\text { - Copiar el enlace del vídeo } \\
\text { - Acceder a la música que utiliza el vídeo para ver más vídeos que } \\
\text { la han utilizado o grabar un vídeo con la misma música. } \\
\text { - Acceder a vídeos que están retransmitiéndose en directo de los } \\
\text { muser a los que sigue la cuenta. }\end{array}$ \\
\hline $\begin{array}{l}\text { MANEJO: Co } \\
\text { y uso del }\end{array}$ & $\begin{array}{l}\text { "publicar vídeos sobre las etiquetas que se ofrecen en la } \\
\text { gescubrir", que es donde están los vídeos agrupados por } \\
\text { g. }\end{array}$ \\
\hline $\begin{array}{l}\text { COMPRENSIC } \\
\text { Ideologías y val }\end{array}$ & $\begin{array}{l}\text { - Cambiar la contraseña } \\
\text { - Añadir correo secundario para recuperar la cuenta } \\
\text { - Ver la lista de bloqueados } \\
\text { - Configurar la cuenta como privada } \\
\text { - Configurar vídeos como privados }\end{array}$ \\
\hline $\begin{array}{r}\text { MANEJO: C } \\
\text { y uso de }\end{array}$ & $\begin{array}{l}\text { - Decidir qué notificaciones push recibir (nuevos seguidores } \\
\text { nuevos me gusta de mi vídeo y nuevo comentarios de mi vídeo) }\end{array}$ \\
\hline
\end{tabular}

Fuente: Elaboración propia.

El análisis comparativo realizado de Musical.ly a TikTok respecto a todas las acciones que ahora permite TikTok (Tabla 3) continúa siendo fundamental para establecer las relaciones y conexiones con las sub competencias de la competencia mediática; 
además, continúa poniendo en evidencia las competencias que los menores necesitan desarrollar en la nueva red social.

\section{CONCLUSIONES Y DISCUSIÓN}

Este artículo pretende continuar con el nuevo campo de investigación abierto por Pérez-Escoda \& Contreras-Pulido (2018), observando cómo evolucionó la Red Social tras su fusión. Al igual que el estudio inicial, adolece de limitaciones metodológicas y de alcance suficiente, que tendrán que ser mejorados en estudios posteriores, aunque este trabajo presenta una continuación del anterior, actualizándolo y adaptándolo a la nueva Red Social.

La actualización de esta investigación tampoco pretendía, como la primera, llegar a conclusiones de gran alcance, pero sí se procuraba observar la evolución en la estructura mediática de la red TikTok según las posibilidades de interacción con respecto a las variables estudiadas, correlacionar las acciones con la competencia mediática actualizando las opciones eliminadas, añadidas y/o modificadas en TikTok, y, finalmente, establecer las nuevas áreas competenciales de la alfabetización mediática y acciones en TikTok.

Se estableció la estructura mediática de TikTok, como se observó en la tabla 1, la cual ha sufrido algunas variaciones en comparación con la estructura mediática de Musical.ly y se puede concluir por los datos obtenidos que TikTok es algo más sencilla que la anterior al simplificar algunos apartados que resultaban anteriormente algo más complejos.

El grado de interacción en Tiktok, como se puede comprobar en el análisis de las cuentas con más seguidores, es muy alto, no solo por el número de seguidores de cada uno de los usuarios, sino también por la interacción con otros seguidores, lo cual se mide por el número de corazones que reciben sus vídeos publicados, cuestión muy generaliza en esta red, donde lo importante es la creación y la copia de los vídeos más populares y la realización de dúos con las cuentas de los usuarios con más seguidores, interactuando entre ellos con el fin de crear una red de interacción entre toda la comunidad muy intensa.

Las acciones de TikTok, en relación con la competencia mediática, se mantuvieron en su mayoría con respecto a Musical.ly, aunque, en cada una de las acciones, algunas opciones fueron eliminadas en su mayoría en las relacionadas con el contenido del perfil y con las herramientas de viralización de los vídeos de los tiktokers con más seguidores. Por lo que se entiende que se continúan "construyendo las lógicas comunicativas" (Pérez-Escoda \& Contreras-Pulido, 2018, p.289).

Se ha podido observar, asimismo, la relación establecida entre las áreas competenciales de la alfabetización mediática y las acciones que se engloban en cada una de las áreas, y cómo ha evolucionado el número de acciones que permite la nueva plataforma. Tras esta actualización de contenido e investigación se concluye, al igual que la investigación anterior, afirmando los beneficios que suponen en el desarrollo competencial en esta nueva Red Social para los menores gracias a que estos pueden "categorizar las acciones que permite en función de unas variables elegidas para el 
estudio y relacionarlas con las competencias de la alfabetización mediática y digital" (Pérez-Escoda \& Contreras-Pulido, 2018, p.289). Y tras analizar la estructura mediática de TikTok, muy similar a la de Musical.Ly, se extraen las mismas conclusiones de que el uso de esta Red Social puede propiciar competencia mediática.

Al prestar atención a las investigaciones llevadas a cabo en los últimos tiempos, son numerosas aquellas que hacen referencia al uso y difusión de las Redes Sociales desde distintos puntos de vista, ya sea desde la implicación activa de los adolescentes en ellas (García-Galera et al., 2014), como de relaciones afectivas y familiares (Padilla et al., 2015; Schrock, 2016; Vidales-Bolaños \& Sábala-Chalezquez, 2017), los vínculos de los jóvenes con la tecnología (Lenhart et al., 2015) o las malas prácticas llevadas a cabo (De-Frutos \& Marcos, 2017; Peddinti et al., 2014; Radesky et al., 2015); sin embargo, cabe destacar que se aprecia una falta de investigaciones encaminadas a dar respuesta a aspectos más pedagógicos de las Redes Sociales en general.

\section{REFERENCIAS}

Aguaded-Gómez, J. I. (2012). La competencia mediática, una acción educativa inaplazable. Comunicar, 20(39), 7 - 8. https://doi.org/10.3916/C39-2012-01-01

Ahlse, J., Nilsson, F., \& Sandström, N. (2020). It's time to TikTok: Exploring generation Z's motivations to participate in \#challenges. Jönpöking University. https://bit.ly/2SfYTB5

Ballesteros-Herencia, C. A. (2020). La propagación digital del Coronavirus: midiendo el engagement del entretenimiento en la Red Social emergente TikTok. Revista Española de Comunicación en Salud, 171-185. https://doi.org/10.20318/recs.2020.5459

Bargh, J. A., \& McKanna, K. Y. (2004). The Internet and social life. Annual Review of $\begin{array}{llll}\text { Psychology, } & 55, & 573 . & -\end{array}$ https://doi.org/10.1146/annurev.psych.55.090902.141922

BBC Mundo (2018, Mayo 2021). Cómo funciona TikTok, la app de selfies de China que se ha convertido en la más descargada de 2018 en iPhones de todo el mundo. https://bbc.in/2QMwHpe

Becerra-Chauca, N., \& Taype-Rondan, A. (2020). TikTok: ¿Una nueva herramienta educativa para combatir la COVID-19? Acta Médica Peruana, 37(2), 249 - 251. https://doi.org/10.35663/amp.2020.372.998

Bernal-Bravo, C., \& Angulo, F. (2013). Interacciones de los jóvenes andaluces en las Redes Sociales. Comunicar, 20(40), 25 - 30. https://doi.org/10.3916/C40-2013-02$\underline{02}$ 
Boyd, D. M., \&. Ellison, N. B. (2007). Social Network Sites: Definition, history, and scholarship. Journal of Computer-Mediated Communication, 13(1), 210 - 230. https://doi.org/10.1111/j.1083-6101.2007.00393.x

Bringué, X., \&. Sádaba-Chalezquer, C. (2011). Menores y Redes Sociales. Foro Generaciones Interactivas. https://bit.ly/3yyNjBV

Buckingham, D., \& Willet, R. (2013). Digital generations: Children, young people, and the New Media. Routledge.

Castañeda-Quintero, L. J., González-Calatayud, V., \& Serrano-Sánchez, J. L. (2011). Donde habitan los jóvenes: precisiones sobre un mundo de Redes Sociales. En F. Martínez, \& I. Solano (Eds.) Comunicación y relaciones sociales de los jóvenes en la Red. (pp.47 - 63). Marfil.

Chan, J. (2019, Junio 5). Top non-game apps by downloads 2018 and 2019. Sensor Tower. https://bit.ly/37cfNDq

Conde, M. A., Forteza-Martínez, A., Andrade-Martínez, C. M. (2020). Análisis de la capacidad de liderazgo y el carisma de los principales youtubers españoles. $3 \mathrm{C}$ TIC. Cuadernos de desarrollo aplicados a las TIC, 9(3), 17 - 41. https://doi.org/10.17993/3ctic.2020.93.17-41

Contreras-Pulido, P., Marfil-Carmona, R., \& Ortega-Tudela, J. M. (2014). La competencia mediática de las personas mayores andaluzas: retos para una inclusión social plena. Historia y Comunicación Social, 19, 129 - 142. https://doi.org/10.5209/rev HICS.2014.v19.44946

Corral, E. M. (2012). El Smartphone como motor de una nueva incertidumbre social: la importancia de las Redes Sociales en la comunicación móvil de los jóvenes españoles en la sociedad de la inmediatez. Prisma Social: Revista de Investigación Social, 8, 87 - 115. https://bit.ly/34c0eeM

Davis, J. (2019). The TikTok strategy: Using Al platforms to take over the world. Knowledge. https://bit.ly/2Tm9MOX

De-Frutos, B., \& Marcos, M. (2017). Disociación entre las experiencias negativas y la percepción de riesgo de las Redes Sociales en adolescentes. El Profesional de la Información, 26(1), 88 - 96. https://doi.org/10.3145/epi.2017.ene.09

Domínguez, D. C. (2010). Las Redes Sociales. Tipología, uso y consumo de las Redes 2.0 en la sociedad digital actual. Documentación de las Ciencias de la Información, 33, 45 - 68. https://bit.ly/3yv8PHy

Fernández, T. (2016). Patrones de uso de las tecnologías de la información y comunicación en estudiantes universitarios. (Tesis Doctoral). Universidad de Granada. https://bit.ly/3vrgvJa 


\section{ESTRUCTURA MEDIÁTICA DE TIKTOK: ESTUDIO DE CASO DE LA RED SOCIAL DE LOS MÁS JÓVENES}

Fernández-de-la-Iglesia, J. C., Casal-Otero, L., Fernández-Morante, C., \& Cebreiro, B. (2020). Actitudes y uso de Internet y Redes Sociales en estudiantes universitarios/as de Galicia: implicaciones personales y sociales. Prisma Social, 28, 145 - 160. https://bit.ly/3rLIIYU

Ferrés, J. (2013). La competencia mediática y emocional de los jóvenes. Revista de estudios de juventud, 101, 89 - 101. https://bit.ly/3bMohoU

Ferrés, J., \&. Piscitelli, A. (2012). La competencia mediática: propuesta articulada de dimensiones e indicadores. Comunicar, 19(38), 75 - 82 https://doi.org/10.3916/C382012-02-08

Gallardo, E. E., Marqués, L., \& Bullen, M. (2014). Usos académicos y sociales de las tecnologías digitales del estudiante universitario de primer año. Tendencias pedagógicas, 23, 191 - 204. https://bit.ly/3tPNCpv

Gallardo, E. E., Marqués, L., \& Bullen, M. (2015). Students in higher education: Social and academic uses of digital technology. RUSC. Universities and Knowledge Society Journal, 12(1), 25 - 37. https://doi.org/10.7238/rusc.v12i1.2078

Gandasegui, V. D. (2011). Mitos y realidades de las Redes Sociales. Información. Y comunicación en la sociedad de la información. Prisma Social, 6, 1 - 26. https://bit.ly/2NBaoBc

García-Galera, M., del-Hoyo-Hurtado, M., \& Fernández-Muñoz, C. (2014). Jóvenes comprometidos en la Red: el papel de las Redes Sociales en la participación social activa. Comunicar, 22(43), 35 - 43. https://doi.org/10.3916/C43-2014-03

Gao, Q., \& Feng, C. (2016). Branding with social media: User gratifications, usage patterns, and brand message content strategies. Computers in Human Behaviour, 63, 868 - 890. https://doi.org/10.1016/i.chb.2016.06.022

García-Ruiz, R., Tirado, R., \& Hernando-Gómez, A. (2018). Redes Sociales y estudiantes: motivos de uso y gratificaciones para el aprendizaje. Aula Abierta, 47(3), 291 - 298. https://bit.ly/3qqwGUB

Gutiérrez-Porlán, I., Román-García, M., \& Sánchez-Vera, M. M. (2018). Strategies for the communication and. Collaborative online work by university students. Comunicar, 26(54), 91 - 99. https://doi.org/10.3916/C54-2018-09

Hermosín-Mojeda, M. J., \&. Conde, M. A. (2015). Competencia sobre el uso de "Smartphones" en menores de edad. Congreso Internacional e Interuniversitario contra la pobreza infantil en el mundo. https://bit.ly/2REg0wv

Herrman, J. (2019). How TikTok is rewriting the world. The New York Times, 10. https://nyti.ms/2SITzMo

Imaña, T. (2009). Facebook, tejiendo la telaraña de las Redes Sociales. Razón y Palabra, 13(1). https://bit.ly/3ucAjy8 
Influencers Marketinghub (2020). Los 18 TikTokers más famosos del planeta. https://bit.ly/2SD8dMx

García-Jiménez, A., Catalina-García, B., \& López-de-Alaya, M. C. (2016). Adolescents and YouTube: Creation, participation, and consumption. Prisma Social, Revista de investigación social, 1, $60-89$. https://bit.ly/3vbPOYH

Katz, J., Rice, R., \&. Acord, S. (2004). Personal mediated communication and the concept of community in theory and practice. En P.J., Kalbfleisch (Ed.), Communication and Community, Communication Yearbook 28 (pp.315 - 371). Erlbaum.

Kautz, H., Selman, B., Shah, M. (1997). Referral web: Combining Social Networks and collaborative filtering. Communications of the ACM, 40(3), $63-65$. https://doi.org/10.1145/245108.245123

Kemp, S. (2019). Digital 2019: Global Internet use accelerates. We are Social. https://bit.ly/3hKZOnO

Lavado, A. (2013). El consumo de YouTube en España. Global Media Journal Mexico, 7(14), 76 - 92. https://bit.ly/3wF594v

Lenhart, A., Smith, A., Anderson, M., Duggan, M., Perrin, A. (2015). Teens, technology, and friendships. Pew Research Center.

Libretilla. (2020). Las 15 cuentas de TikTok con más seguidores. https://bit.ly/3I6Uw4k

Lim, L., Leong-Weng, L., Yen, L. W., Jie, H., \& Simandjoentak, J. P. (2019). Bytedance: The road to success of a global influential social media platforms creator. Academy of Asian Business Review, 5(2), 77 - 100. https://bit.ly/3bMgk3e

Liqian, H. (2018). Study on the perceived popularity of TikTok. Bangkok University. https://bit.ly/3oCwOjg

Literat, I., \& Kligler-Vilenchik, N. (2019). Youth collective political expression on social media: The role of affordances and memetic dimensions for voicing political views.

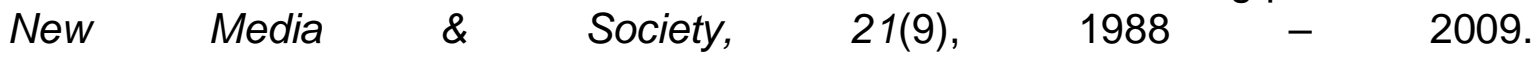
https://doi.org/10.1177/1461444819837571

Livingstong, S. (2002). Young people and new media: Childhood and the changing media environment. Sage.

Marcelino-Mercedes, G. V. (2015). Migración de los jóvenes españoles en Redes Sociales, de Tuenti a Facebook y de Facebook a Instagram. La segunda migración. Revista de comunicación y tecnologías emergentes, 13(2), 48 - 72. https://doi.org/10.7195/ri14.v13i2.821 


\section{ESTRUCTURA MEDIÁTICA DE TIKTOK: ESTUDIO DE CASO DE LA RED SOCIAL DE LOS MÁS JÓVENES}

Marín-Gutiérrez, I., Díaz-Pareja, E., \& Aguaded-Gómez, J. I. (2013). La competencia mediática en niños y jóvenes: la visión de España y Ecuador. Chasqui. Revista Latinoamericana de comunicación, $124, \quad 41 \quad$ - 47. https://doi.org/10.16921/chasqui.v0i124.17

Muñoz-Saldaña, M., Sádaba, C., \& Naval, C. (2011). La competencia mediática en los adolescentes, el caso de Internet. Estrategias de Alfabetización Mediática: Reflexiones sobre comunicación y educación, 1 - 16. Editorial UOC.

Olivares-García, F. J., \& Méndez-Majuelos, I. (2020). Análisis de las principales tendencias aparecidas en TikTok durante el periodo de cuarentena por la COVID19. Revista Española de Comunicación en Salud, 243 - 252. https://doi.org/10.20318/recs.2020.5422

Orihuela-Colliva, J. L. (2008). Internet: la hora de las Redes Sociales. Nueva Revista de política, cultura y arte. 119, 57 - 65. https://bit.ly/3woFXik

Padilla, S., Rodríguez, E., Álvarez, M., Torres, A., Suárez, A., \& Rodrigo, M. J. (2015). La influencia del escenario educativo familiar en el uso de Internet en los niños de primaria y secundaria. Infancia y aprendizaje, 38(2), 402 - 434. https://doi.org/10.1080/02103702.2015.1016749

Peddinsti, S. T., Ross, K. W., \& Cappos, J. (2014). On the Internet, nobody knows you're a dog: A Twitter case study of anonymity in social networks. Proceedings of the Second ACM Conference on Online Social Networks. (pp.83 - 94). ACM.

Pedrero-Pérez, E. J., Ruiz-Sánchez-de-León, J., Rojo-Mota, G., Llanero-Luque, M., Pedrero-Aguilar, J., Morales-Alonso, S., \& Puerta-García, C. (2018). Tecnologías de la Información y la Comunicación (TIC): uso problemático de Internet, videojuegos, teléfonos móviles, mensajería instantánea y Redes Sociales mediante el MULTICAGE-TIC. Adicciones, 30(1), 32. https://doi.org/10.20882/adicciones.806

Peña, M. A., Rueda, E., \& Pegalajar, M. C. (2018). Posibilidades didácticas de las Redes Sociales en el desarrollo de competencias de educación superior: percepciones del alumnado. Píxel-Bit. Revista de medios y educación, 53, 239 252. https://doi.org/10.12795/pixelbit.2018.i53.16

Pérez-Escoda, A., \& Contreras-Pulido, P. (2018). Smartphone y Redes Sociales para el desarrollo de competencias mediáticas y digitales en niños y adolescentes: Musical.ly. Aula Abierta, 47(3), $281 \quad$ - 290. https://doi.org/10.17811/rifie.47.3.2018.281-290

Pérez-Rodríguez, A., \& Delgado-Ponce, Á. (2012). De la competencia digital y audiovisual a la competencia mediática: dimensiones e indicadores. Comunicar, 20(39), 25 - 33. https://doi.org/10.3916/C39-2012-02-02 
Radensky, J. S., Schumacher, J., \& Zuckerman, B. (2015). Mobile and interactive media use by young children: The good, the bad, and the unknown. Pediatrics, 135(1), 1 - 3. https://doi.org/10.1542/peds.2014-2251

Rettberg, J. W. (2017). Hand sings for lop-syncing: The emergence of a gestural language on Musical.ly as a video-based equivalent to emoji. Social Media Society, 3(4), 2056305117735751. https://doi.org/10.1177/2056305117735751

Robehmed, N. (2017, 11 de mayo). From musers to money: Inside video app Musical.ly's coming of ages. Forbes. https://bit.ly/2LxfOeN

Rodrigo-Alsina, M. (2001). Teorías de la comunicación: ámbitos, métodos y perspectivas. Servei de Publicacions de la Universitat Autònoma de Barcelona.

Ros-Martín, M. (2009). Evolución de los servicios de Redes Sociales en Internet. El profesional de la Información, 18(5), $552 \quad$ - 558. https://doi.org/10.3145/epi.2009.sep.10

Rostaminezhad, M. A., Porshafei, H., \& Ahamdi, A. A. (2019). Can effective study approaches mediate the negative effect of social networking on academic performance? Education and Information Technologies, 24(1), 205 - 217. https://doi.org/10.1007/s10639-018-9770-y

Santos, E. (2016, 5 de noviembre). Musical.ly: qué es, cómo funciona y por qué tiene tanto éxito entre los adolescentes. Xataka. https://bit.ly/3hJgufl

Schrock, A. R. (2016). Exploring the relationship between mobile Facebook and Social Capital: What is the "Mobile difference" for Parents of Young Children? Social Media \& Society, 2(3). https://doi.org/10.1177/2056305116662163

Smith, C. (2019, 12 de marzo,). 40 douyin, TikTok, Musical.ly statistics and facts 2019. DMR Statistics. https://bit.ly/3oGHpKb

Solís, A. (2019, 1 de febrero). Así es TikTok: la cuarta app más descargada en España. ED Economía Digital. https://bit.ly/3uaWkO5

Sotelo, R. (2018). Adiós Musical.ly, hola TikTok: la app de vídeo corto para adolescentes se integra en TikTok y causa la furia de los musers. Marketing 4 Ecommerce. https://bit.ly/3vgJKye

Stokel-Walker, C. (2019). Chinese app TikTok may be leaking users' data. But some of the privacy concerns also apply to apps developed in Silicon Valley, finds Chriss Stokel-Walker. New Scientist, 244(3260), 14. https://doi.org/10.1016/s0262$\underline{4079(19) 32341-3}$

Subrahmanyam, K., Reich, S. M., Waechter, N., \& Espinoza, G. (2008). Online and offline Social Networks: Use of social networking sites by emerging adults. Journal of Applied Developmental Psychology, 29(6), 420 - 433. https://doi.org/10.1016/j.appdev.2008.07.003 
Tabernero, C., Juárez, D. A., \& Navarro, J. S. (2010). Juventud y tecnologías digitales: espacios de ocio, participación y aprendizaje. Revista de estudios de juventud, 8, 77 - 96. https://bit.ly/3yz0pPm

Telefónica (2009). La generación interactiva en España. Fundación Telefónica. https://bit.ly/3fTyqld

Vidales-Bolaños, M., \& Sábada-Chalezquer, C. (2017). Adolescentes conectados: la medición del impacto del móvil en las relaciones sociales desde el capital social. Comunicar, 25(53), 19 - 28. https://doi.oorg/10.3916/C53-2017-02

Wang, Y. (2020). Influence of camera view on TikTok users' presence, immersion, and adoption intent. Computers in Human Behavior, 110, 196373. https://doi.org/10.1016/i.chb.2020.106373

Wang, R. (2019). Effect of e-wom message of opinion leaders on purchase intention of female consumers in China: Case of Ddouyin (Tik Tok). Instituto Superior de Economia e Gestão. https://bit.ly/3vbNjWo

Xataka. (2018). Así es TikTok, la app más descargada de iOS en lo que llevamos de 2018. https://bit.ly/3oRUEYV

Yang, S., Zhao, Y., \& Ma, Y. (2019). Analysis of the reasons and development of short video application - Taking Tik Tok as an example. Proceedings of the $20199^{\text {th }}$ International Conference on Information and Social Science (ICISS 2019). https://bit.ly/3oHN1DW

Zhou, Q. (2019). Underrstanding user behaviours of creative practice on short video sharing platforms. A case study of TikTok and Bilibili. (Tesis Doctoral). Universidad de Cincinnaty. https://bit.ly/3f7lXeh

\section{AUTORES}

\section{Manuel Antonio Conde del Rio}

Doctorando del Programa de Doctorado Interuniversitario en Comunicación de las universidades de Cádiz, Huelva, Málaga y Sevilla, en la línea de Educomunicación y Alfabetización Mediática (Media Literacy). Máster en Comunicación y Educación Audiovisual por UNIA/UHU, y Grado en Educación Social por la Universidad de Huelva.

Orcid ID: https://orcid.org/0000-0002-7382-2166

Google Scholar: https://scholar.google.es/citations?hl=es\&user=l2RtBIQAAAAJ

ResearchGate: https://www.researchgate.net/profile/Manuel Conde 